\title{
Identification and Characterization of CYC-Like Genes in Regulation of Ray Floret Development in Chrysanthemum morifolium
}

\author{
Di Huang, Xiaowei Li, Ming Sun, Tengxun Zhang, Huitang Pan, Tangren Cheng, Jia Wang \\ and Qixiang Zhang *
}

Beijing Key Laboratory of Ornamental Plants Germplasm Innovation and Molecular Breeding, National Engineering Research Center for Floriculture, Beijing Laboratory of Urban and Rural Ecological Environment, Key Laboratory of Genetics and Breeding in Forest Trees and Ornamental Plants of Ministry of Education, School of Landscape Architecture, Beijing Forestry University, Beijing, China

OPEN ACCESS

Edited by:

Catherine Anne Kidner,

University of Edinburgh, UK

Reviewed by:

Minsung Kim,

University of Manchester, UK

Beth Thompson,

East Carolina University, USA

${ }^{*}$ Correspondence:

Qixiang Zhang

zqxbjfu@126.com

Specialty section:

This article was submitted to Plant Evolution and Development,

a section of the journal

Frontiers in Plant Science

Received: 19 May 2016

Accepted: 17 October 2016

Published: 07 November 2016

Citation:

Huang $D, L i X$, Sun $M$, Zhang $T$,

Pan $H$, Cheng $T$, Wang $J$ and

Zhang Q (2016) Identification and Characterization of CYC-Like Genes in Regulation of Ray Floret

Development in Chrysanthemum morifolium. Front. Plant Sci. 7:1633.

doi: 10.3389/fpls.2016.01633
Chrysanthemum morifolium, one of the most economically important ornamental crops worldwide, is well-known for the elaborate and complex inflorescence which is composed of both bilaterally symmetrical ray florets and radially symmetrical disc florets. Despite continuing efforts, the molecular mechanisms underlying regulation of the two flower types are still unclear so far. CYC-like proteins have been shown to control flower symmetry or regulate flower-type identity in several angiosperm plant lineages. In this study, we conducted comparative analysis of the $\mathrm{CmCYC2}$ genes in two chrysanthemum cultivars and their F1 progenies with various whorls of ray florets. Six CmCYC genes were identified and sequenced, all of which were grouped into the CYC2 subclade. All the six $\mathrm{CmCYC2}$ genes were predominantly expressed in reproductive organs, and in particular in the petal of ray florets. Of these genes, the transcription level of $\mathrm{CmCYC2C}$ was highly up-regulated in ray florets of the double-ray flowered heads. In addition, the result that $\mathrm{CmCYC2C}$ was highly expressed at key developing stages indicates its role in regulating petal development. Furthermore, overexpression

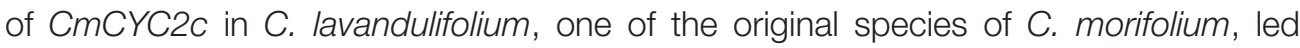
to significant increase in flower numbers and petal ligule length of ray florets. Besides $\mathrm{CmCYC2c}$, the expression of $\mathrm{CmCYC2f}$ was also significantly up-regulated in transgenic lines, implying a possible role in regulating development of ray florets. Both results of expression patterns and transgenic phenotypes suggest that $\mathrm{CmCYC2c}$ is involved in regulating ray floret identity in the chrysanthemum. This study will be useful for genetic manipulation of flower shape in chrysanthemum and hence promote the process of molecular breeding.

Keywords: Chrysanthemum morifolium, CYCLOIDEA, TCP, inflorescence, flower symmetry, flower development

\section{INTRODUCTION}

Asteraceae is characterized for a highly compressed capitulum which superficially resembles a large single flower but is composed of numerous individual flowers (Funk, 2009). These flowers are divided into two types: ray and disc florets (DF; Figure 1). The peripherally located ray florets are bilaterally symmetrical with two rudimentary dorsal petals (dp) and elongated ventral ligule (vl) 


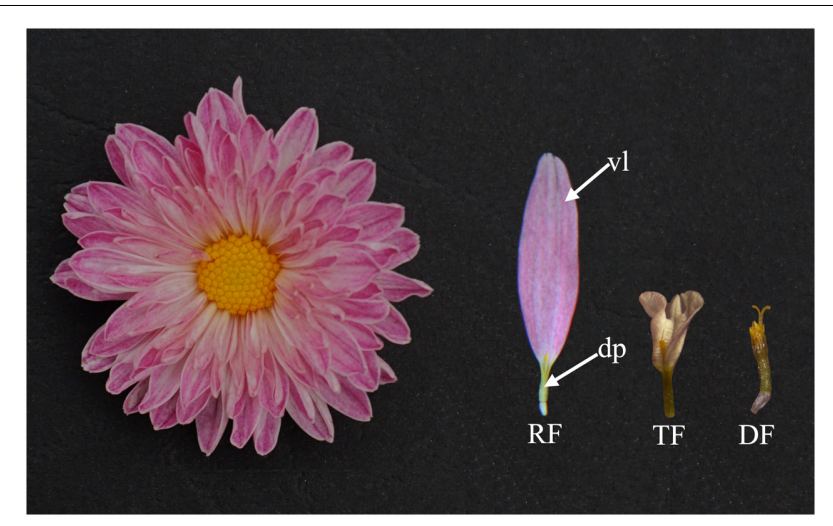

FIGURE 1 | Inflorescence of Chrysanthemum morifolium. The marginal ray florets (RF) are female and bilaterally symmetrical. The central disc florets (DF) are hermaphrodite and bear carpel and pollen-producing stamens. The ray florets have showy ventral ligule (vl) formed by three fused petals while the two dorsal petals (dp) are rudimentary. Some chrysanthemum cultivars have intermediate trans florets (TF) similar to ray florets but with smaller or abnormal petal ligule.

formed by three fused petals. Instead, the central DF are radially symmetrical with five equivalent petals (Bremer and Anderberg, 1994). The ray florets are generally female with arrested stamens, whereas the DF develop pollen-producing stamens in addition to carpels (Bremer and Anderberg, 1994; Gillies et al., 2002). Besides ray and $\mathrm{DF}$, some chrysanthemum cultivars have a third flower type: trans floret (TF) which is morphologically similar to ray floret but with smaller or abnormal ventral petal ligule (Figure 1). Different combinations of these florets and variation of the petal types give rise to a variety of flower head types. Moreover, the presence of the showy ray florets in the capitulum has been shown to be associated with pollinator-mediated speciation, outcrossing rage and genetic diversity, and may lead to the evolutionary success of the Asteraceae (Marshall and Abbott, 1984; Sun and Ganders, 1990; Endress, 1999; Sargent, 2004; JuntheikkiPalovaara et al., 2014). Both classical and modern molecular genetic studies have indicated that the presence or absence of ray florets is mainly under the control of one or two major genes and some other modifier genes (Gillies et al., 2002; Andersson, 2008).

The TCP family of transcription factors has been shown to participate in regulating floral symmetry in a range of species (Luo et al., 1996, 1999; Cubas et al., 1999b, 2001; Kim et al., 2008; Yang et al., 2012). Proteins encoded by members of the TCP family share a conserved basic helix-loop-helix TCP domain (Cubas et al., 1999a; Aguilar-Martínez et al., 2007). The TCPs are divided into two classes: PCF and CYC/TB1. The CYC/TB1 lineage is specific of angiosperms and before the radiation of the core eudicots, it has experienced gene duplications, which give rise to three subclades, CYC1, CYC2, and CYC3 (Howarth and Donoghue, 2006). The studies in Antirrhinum majus show that the two CYC2 clade genes CYC and its paralog DICHOTOMA $(D I C H)$ are involved in flower symmetry regulation via specific expression in the dorsal petals of the flower and arresting the development of the dorsalmost stamen (Luo et al., 1996, 1999; Cubas et al., 1999b; Costa et al., 2005). The peloric radial symmetrical mutants of both Linaria vulgaris and A. majus are caused by the loss of expression of $L v C Y C$ and $C Y C$ via extensive DNA methylation or transposon insertion, respectively (Luo et al., 1996; Cubas et al., 1999b). Increasing evidences indicate that members of the CYC2 subclade have been repeatedly recruited to function in the control of floral zygomorphy during evolution. Similar to the adaxialized mutant in Antirrhinum caused by ectopic expression of CYC, the radial flowers of Cadia purpurea are dorsalized and all petals have acquired dorsal identity as a result of $\operatorname{LegCYC}$ expression in all five petals (Citerne et al., 2006). Meanwhile, LjCYC2 from Lotus japonicas has also been found to be important in the establishment of dorsal identity (Feng et al., 2006). Furthermore, studies in Arabidopsis and Primulina heterotricha suggest that the persistent expression of CYC-like genes in later developmental stages is important for the development of corolla zygomorphy (Cubas et al., 2001; Yang et al., 2012). For example in P. heterotricha, two CYC2 clade proteins, CYC1C and CYC1D, have been shown to positively auto-regulate themselves and cross-regulate each other to maintain dorsally restricted gene expression and dorsoventral differentiation in zygomorphic flowers (Yang et al., 2012). Therefore, the alteration of flower symmetry and modification of flower morphology (mainly for the dorsal petals) are usually caused by the spatial-temporal expression variation of the CYC2 subclade genes (Yang et al., 2015).

An independently CYC2-mediated pathway has apparently been recruited for inflorescence development in Asteraceae. Instead of mainly expressed in the dorsal portion to establish bilateral symmetry of individual flower, CYC-like proteins in Asteraceae participate in regulating the identity of flower types in inflorescence (Broholm et al., 2008; Kim et al., 2008; Juntheikki-Palovaara et al., 2014). In sunflower, the turf or tub mutants are characterized by a shift from zygomorphic to actinomorphic ray floret, due to insertion of transposable elements in the HaCYC2c TCP motif that leads to a premature stop codon (Fambrini et al., 2011, 2014a; Chapman et al., 2012). In contrast, in the $d b l$ or chry mutants, caused by an insertion upstream the coding region, $\mathrm{HaCYC} 2 \mathrm{c}$ is expressed throughout the flower head, converting DF into ray-like ones (Chapman et al., 2012). However, phenotypic analysis in $F_{2}$ and $\mathrm{F}_{3}$ progenies derived from the crosses Chry $2 \times$ turf demonstrates that the CACTA insertion is not always sufficient to change the expression of $\mathrm{HaCYC} 2 \mathrm{c}$ gene and produce Chry 2 phenotype (Fambrini et al., 2014b). Ectopic expression of Gerbera CYClike genes in transgenic Gerbera leads to similar changes with elongated petals and disrupted stamen development in DF (Broholm et al., 2008). Furthermore, it is possible that the context-specific protein complex involving GhCYC2 proteins and their co-regulators may target different downstream genes (Juntheikki-Palovaara et al., 2014). Unlike in Gerbera, RAY1, $R A Y 2$, and RAY3 in Senecio are only involved in promoting ventral identity in ray florets, whereas no change is found in DF (Kim et al., 2008; Chapman and Abbott, 2010; Garcês et al., 2016). Therefore, although a great amount of data have shown that these CYC-like genes in Asteraceae are key regulators in regulating flower development, a much more complex regulatory system seems to stay behind the complex 
flower head (Fambrini et al., 2014b; Juntheikki-Palovaara et al., 2014).

Chrysanthemum morifolium, one of the 10 most popular Chinese traditional flowers, is a typical example of radiate species with inflorescence composed of both zygomorphic and actinomorphic symmetric flowers (Teixeira da Silva, 2003; Teixeira da Silva et al., 2013; Qi et al., 2016). The contemporary chrysanthemum cultivars are characterized by the substantial variation in petal types and the complicated ploidy levels (from $2 \mathrm{n}=4 \mathrm{x}=36$, to $6 n=54,72$, up to 90) (Liu et al., 2012). The allopolyploid and self-incompatible traits of chrysanthemum make it difficult to investigate the mechanism underlying regulation of the complex inflorescence. Recently, CYC-like TCP domain proteins have been shown to regulate morphological novelties during plant evolution (Luo et al., 1999; Kim et al., 2008; Hileman, 2014). In this study, two hexaploid chrysanthemum cultivars and several of their F1 progenies were selected to investigate the possible role of the chrysanthemum CYC-like genes in establishing the complex inflorescences. A total of six CYC2 genes were amplified from C. morifolium. The expression patterns of these genes were then compared during early inflorescence development stages and among several different floral heads with various whorls of ray florets. The function of $\mathrm{CmCYC2c}$, which was remarkably up-regulated in the doubleray flowered heads (with multiple whorls of ray florets), was further characterized via overexpression in a wild diploid specie C. lavandulifolium. The growth of ray florets was significantly promoted in the positive transgenic lines. Meanwhile, the expression of CmCYC2f was also remarkably up-regulated. Hence, we speculate that the CYC2 subclade in chrysanthemum has expanded during evolution, and in particular, the $\mathrm{CmCYC2c}$ of these genes is capable of regulating the growth of ray florets.

\section{MATERIALS AND METHODS}

\section{Plant Materials and Growth Conditions}

Two cultivars of C. morifolium and their F1 progenies (Figure 2A), as well as the wild diploid species C. lavandulifolium were grown in the standard experimental fields at Xiao Tang Shan, affiliated to Beijing Forestry University, Beijing, China. C. morifolium 'Guoqing xiaoliuhao' (GQ) and 'Mao xiangyu' (MXY) (Figure 2B) are two hexaploid $(2 n=54)$ ground-cover chrysanthemum cultivars with different whorls of ray florets. C. lavandulifolium $(2 n=18)$ used for plant transformation is one of the original species of $C$. morifolium with relatively simple genetic background. Wild-type (WT) and transgenic plantlets of C. lavandulifolium with six leaves were transplanted into flowerpots containing a mixture of turf and vermiculite substrate $(\mathrm{V}: \mathrm{V}=1: 1)$. All these plantlets were managed routinely in the standard greenhouse under a 16 -h light/8-h dark cycle for 150 days, and then an 8 -h light/16-h dark cycle at $25 \pm 2^{\circ} \mathrm{C}$ with $\sim 55 \%$ relative humidity.

\section{Isolation of CmCYC2 Subclade Genes}

Total RNA was extracted from young inflorescences of C. morifolium 'Guoqing xiaoliuhao', 'Mao xiangyu', and
C. lavandulifolium using the TRIzol reagent (Invitrogen, USA) according to the manufacturer's instructions. The RNA was treated with RQ1 RNase-free DNase (Promega, USA) according to the manufacturer's protocol to remove residual genomic DNA. The RNA integrity was analyzed by gel electrophoresis, and its concentrations were measured and equalized within sample sets. The first strand of cDNA was synthesized based on $2 \mu \mathrm{g}$ of total RNA using the M-MLV reverse transcription system (Promega, Madison, WI, USA). The TCP and R domain factors of CYC2 subclade genes were amplified using one pair of degenerate primers designed corresponded to the amino acid sequences ASKTLDWL and RARARERT of available GenBank Asteraceae sequences (accession number: FJ356704.1, FJ356700.1, EU429303.1, EU429304.1, and EU088368-EU088372). PCR conditions were the following: $94^{\circ} \mathrm{C}$ for $30 \mathrm{~s}, 54^{\circ} \mathrm{C}$ for $30 \mathrm{~s}$, and $72^{\circ} \mathrm{C}$ for $1 \mathrm{~min}$ for 30 cycles. Multiple clones were sequenced and analyzed in an attempt to find all the CYC-like paralogs. The full length cDNAs were amplified by using the SMART RACE cDNA amplification kit (Clontech, Japan) to carry out $3^{\prime}$ and $5^{\prime}$ RACE (rapid amplification of cDNA ends). All of the amplified products were sub-cloned into the pMD18-T vector (Takara, Japan) and transformed into E. coli $\mathrm{DH} 5 \alpha$ for sequencing. All primers were listed in Supplementary Table S1. The mRNA coding sequences of these genes have been uploaded to the GenBank database (accession numbers KU595426-KU595431 and KX161379-KX161384).

\section{Sequence Alignment and Phylogenetic Analyses}

Multiple alignment of CYC/TB1-like protein sequences from C. morifolium and the other selected eudicots species were performed using ClustalX 2.0 with default parameters (Larkin et al., 2007), and the BioEdit software (version 7.1) was used to edit the aligned sequences. Protein sequences between the TCP and $\mathrm{R}$ domains were selected to generate maximum likelihood (ML) tree using PhyML (Guindon et al., 2010) under the JonesTaylor-Thornton (JTT) model with 100 bootstrap replicates. Numbers above branches indicate local bootstrap value ( $>50 \%$ support). Prediction of conserved motifs of $\mathrm{CmCYC2}$ clade genes was performed using the MEME online tool ${ }^{1}$.

\section{Scanning Electron and Light Microscopy}

The young developing flower heads of MXY at different stages were collected and fixed overnight in FAA, and then dehydrated through a gradient ethanol series into $100 \%$ after being dissected under a stereomicroscope ethanol. After being chemically dried by washing in a 2-Methyl-2-propanol series and air-dried under vacuum, the samples were mounted on aluminum stubs and sputter coated with gold. Photographs were taken by a Hitachi S$3400 \mathrm{~N}$ scanning electron microscope (SEM) in Beijing Forestry University.

The flower buds in $100 \%$ ethanol (procedures before this were the same as that mentioned above for SEM) were moved into a gradient xylene series. Then, the samples were embedded in paraffin and cut into $8 \mu \mathrm{m}$ sections. For histological staining,

${ }^{1}$ http://meme-suite.org/tools/meme 


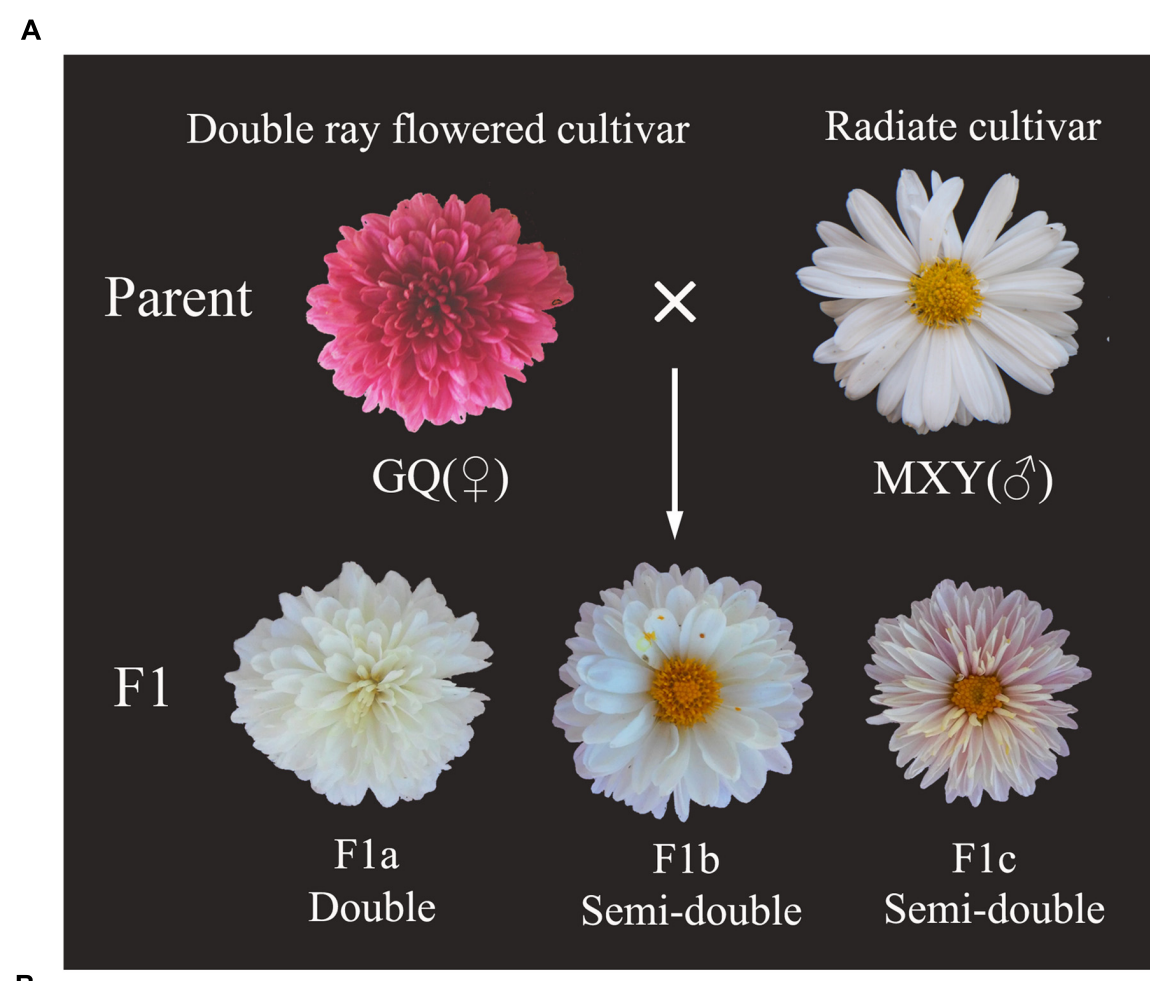

B
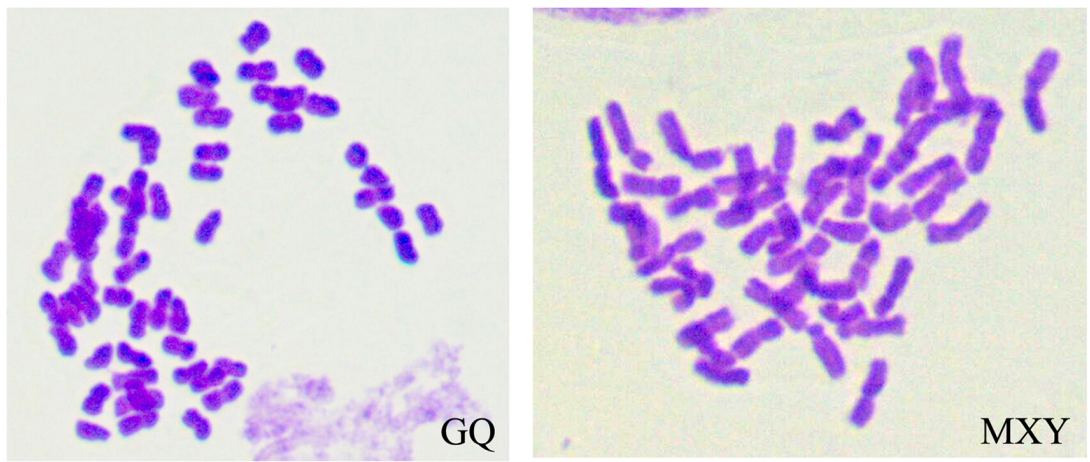

FIGURE 2 | Flower morphologies and chromosome numbers of several C. morifolium lines with various whorls of ray florets. (A) Inflorescence of two chrysanthemum cultivars and their F1 progenies have intermediate whorls of ray florets compared with that of two parents. Cultivar abbreviations are as follows: GQ, C. morifolium 'Guoqing xiaoliuhao'; MXY, C. morifolium 'Mao xiangyu.' (B) Chromosome numbers of the two chrysanthemum parents $(G Q$ and MXY, $2 n=6 x=54)$.

paraffin was removed with xylene and the sections were stained with safranin ( $1 \%$ in water) and fast green $(0.3 \%$ in $95 \%$ ethanol). The sections were observed and photographed under a light microscope (Zeiss Axio scope. A1) in Beijing Forestry University.

\section{Quantitative Real-Time PCR Analyses}

Quantitative RT-PCR (qRT-PCR) assay was used to detect the expression levels of the $\mathrm{CmCYC2}$ genes in inflorescence primordia of MXY at six early developmental stages (stages I-III, Figure 3) and one late stage (stage IV) at which the petal ligules of ray florets began to expand (Supplementary Figure S1). To analyze the specific expression patterns of the $\mathrm{CmCYC2}$ genes in ray and DF of C. morifolium, the doubleray flowered cultivar GQ and the radiated cultivar MXY, as well as two of their F1 progenies (F1a and F1b) with intermediate whorls of ray florets were prepared (Figure 2A). Each sample was a pooling of six to eight inflorescences at later developing stages (pooled from stage 3, 4, 6, 7, 8, and 9) (Supplementary Figure S1). We defined the later inflorescence developmental stages comparable to those defined in Gerbera (Bradley et al., 1993; Laitinen et al., 2007). In addition, the disc floret samples were only excised from their centermost florets while the ray floret samples were a mix of flowers from every whorl. For tissue-specific expression analysis, vegetative and reproductive organs (a mixture of stage 8 and 9) were dissected from another F1 progeny (F1c) which developed some trans-like florets between the normal ray and disc ones (Figure 2A). Additionally, the stamen sample was pooled from DF only, and the ovary plus stigma and style sample was a 
A

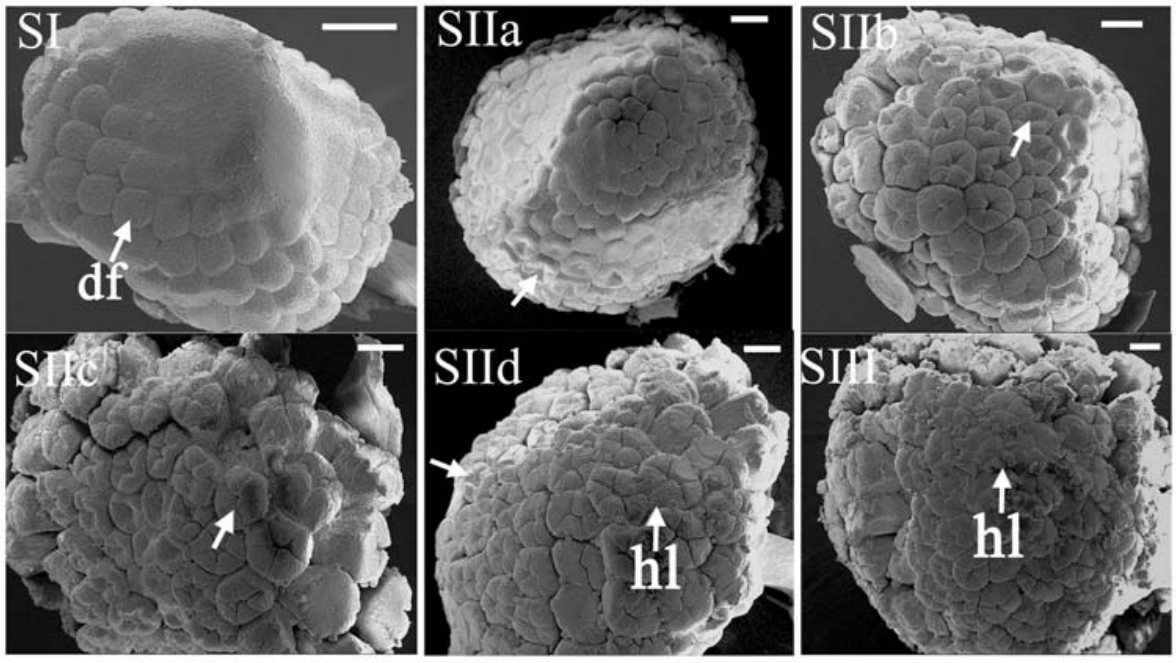

B
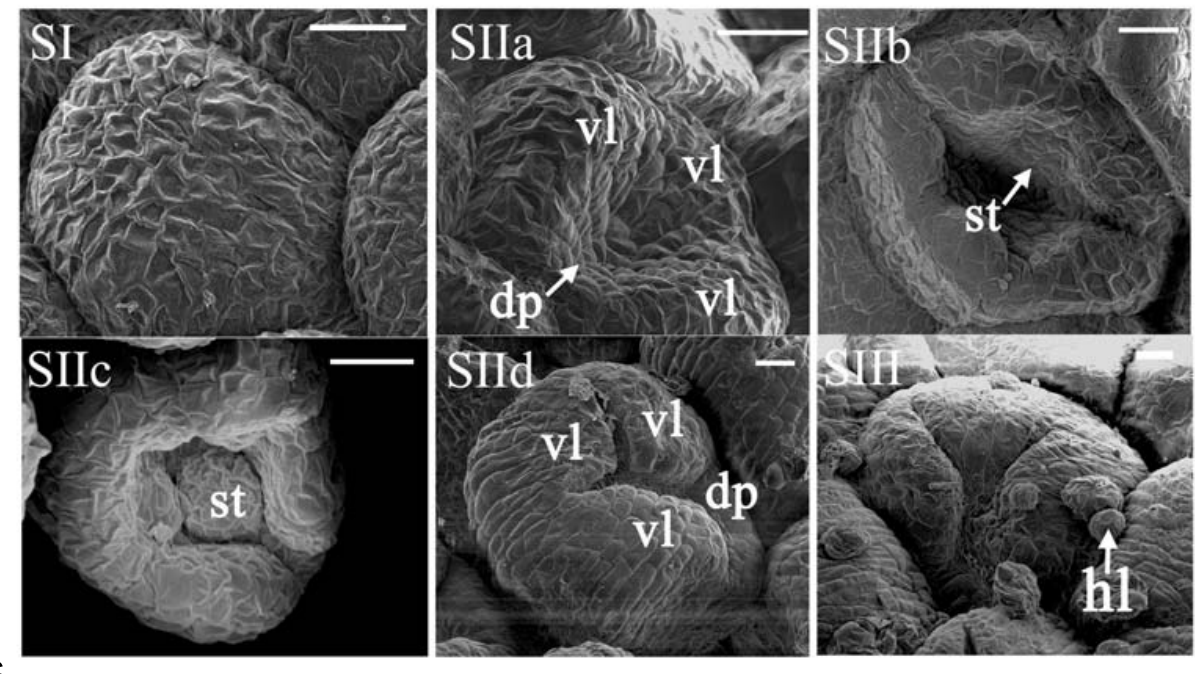

C

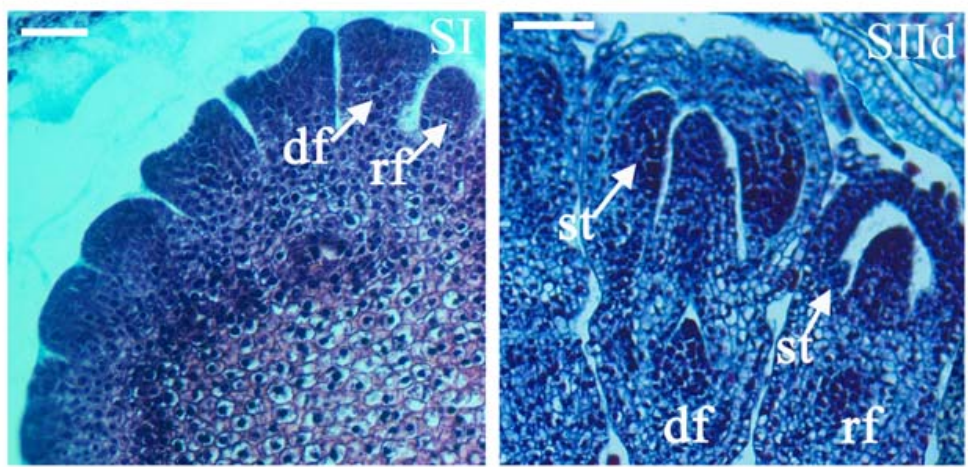

FIGURE 3 | Morphological analysis of the early development stages of ray and DF in C. morifolium 'Mao xiangyu' (MXY). (A) SEM images show developing capitula (stages I-III). The primordia of the disc florets (df) develop acropetally from the basal to the upper during the whole ontogeny process. The ring-shaped petal primordia began to form at stage llb (arrowed) for the inner whorls of DF, whereas the peripheral whorls of DF already had stamen primordia at stage Ila (arrowed). The petal of peripheral DF finally covered the inner organs at stage Ilc (arrowed). At stage Ild, nearly all the DF finished organ differentiation except for the innermost ones (arrowed). Hair like (hl) structures began to emerge at stage lld. Scale bars: $100 \mu \mathrm{m}$. (B) SEM images show developing ray florets (stages I-III). Asymmetry growth of petals in ray florets started to emerge at stage lla with accelerated elongation of three ventral petal ligules (VI) and ceased growth of two dorsal petals (dp). The stamens and two dorsal petals gradually ceased growth while the ventral petal ligule continued to elongate during stage IIc-III. Scale bars: $20 \mu \mathrm{m}$. (C) Histological analysis of ray (rf) and disc floret (df) primordia at stage I and II d. The primordia of the ray florets (arrowed) appeared to be smaller than those of the disc ones at stage I. The development of rudimentary stamens (st) in ray florets lagged behind at stage Ild. Scale bars: $50 \mu \mathrm{m}$. 
mixture of all the three types of florets. All plant materials collected were immediately placed in liquid nitrogen and stored at $-80^{\circ} \mathrm{C}$ until extraction. Three biological replicates were collected for each sample. The extraction of total RNA and the synthesis of the cDNA were performed as described above. The cDNA sample was diluted five times with $80 \mu \mathrm{l}$ of deionized water for all gene expression analyses. The $\mathrm{qPCR}$ reactions were performed using a Mini Opticon Real-time PCR System (Bio-Rad, USA) with SYBR Premix Ex TaqII kit (TaKaRa, Japan) following the manual's recommendations. All gene-specific primer pairs for qRT-PCR were listed in Supplementary Table S1, and their amplification efficiencies were analyzed to make sure that they were closed to $100 \%$. The PCR program was conducted with an initial step of $30 \mathrm{~s}$ at $95^{\circ} \mathrm{C}$, followed by 40 cycles at $95^{\circ} \mathrm{C}$ for $5 \mathrm{~s}, 58^{\circ} \mathrm{C}$ for $30 \mathrm{~s}$, and $72^{\circ} \mathrm{C}$ for $30 \mathrm{~s}$. Product specificity for each primer pair was verified by melting-curve analysis. The relative expression levels were calculated using the $2^{-\Delta \Delta} C t$ method, with CnActin (GenBank accession number KF305683.1) gene as the endogenous control.

\section{Vector Construction and Plant Transformation}

The ORF of $C m C Y C 2 c$ was obtained by PCR amplification using gene-specific primers $\mathrm{CmCYC} 2 c-\mathrm{F} 2$ and $\mathrm{CmCYC} 2 c-\mathrm{R} 2$ (both containing NcoI enzyme site). Driven under the control of the CaMV $35 \mathrm{~S}$ promoter, the $\mathrm{CmCYC2c}$ was cloned into a modified binary pCOMBIA1304 vector. Then the 35S:: $\mathrm{CmCYC2c}$ plasmid was transformed into C. lavandulifolium by the Agrobacterium tumefaciens EHA105-mediated freezethaw method (Chen et al., 2004). For transformation, small leaf pieces with a diameter less than $8 \mathrm{~mm}$ were cut from newly formed expanding leaves of C. lavandulifolium using scalpels. After $48 \mathrm{~h}$ pre-culture on MS medium, these leaves were soaked in the Agrobacterium bacteria solution containing $20 \mu \mathrm{M}$ acetosyringone (final $\mathrm{OD}_{600}=0.4-0.6$ ) for $10 \mathrm{~min}$, with excess broth sucked away by sterilized absorbent paper. Then the leaf pieces were cultured on the CI (callus-induction) medium (MS medium $+1.0 \mathrm{mg} \mathrm{l}^{-1}$ 6-BA, $0.5 \mathrm{mg}^{-1} \mathrm{NAA}$ ) for 3 days in darkness. After being washed with sterilized water, the explants were placed on the bacteria elimination medium (CI medium containing $400 \mathrm{mg} \mathrm{l}^{-1}$ carbenicillin) for 5 days, and then were transferred to the selection medium I (CI medium containing $400 \mathrm{mg} \mathrm{l}^{-1}$ carbenicillin and $8 \mathrm{mg}$ $\mathrm{l}^{-1}$ hygromycin) for the selection of putatively transformed calli. After two subcultures, the explants were placed on the selection medium II (containing only $200 \mathrm{mg} \mathrm{l}^{-1}$ carbenicillin) to promote callus differentiation. The medium was replaced every 2 weeks until the regenerated plantlets grow up with 2-4 leaves. Then the putatively transformed plantlets were transferred to the rooting medium (MS medium $+10 \mathrm{mg} \mathrm{l}^{-1}$ hygromycin, $200 \mathrm{mg} \mathrm{l}^{-1}$ carbenicillin). All the materials were cultured at the same conditions with $16 \mathrm{~h}$ light $/ 8 \mathrm{~h}$ dark at $24 \pm 2^{\circ} \mathrm{C}$. The transgenic plants selected from hygromycin were verified using a pair of CaMV $35 \mathrm{~S}$ promoter-specific forward primer and $\mathrm{CmCYC} 2 \mathrm{c}$-specific reverse primer (Supplementary Table S1).

\section{Gene Expression Analysis and Phenotypic Observation of Transgenic Lines}

RNA extraction and cDNA synthesis were performed as described above from full opened inflorescence. We used two primer pairs to distinguish the expression levels of the transgene $(\mathrm{CmCYC} 2 c)$ and the endogenous gene $(\mathrm{ClCYC} 2 c)$ in full opened inflorescences of six positive transgenic lines. One specific primer pair $\mathrm{CmCYC} 2 c-\mathrm{F} 1 / \mathrm{CmCYC} 2 c-\mathrm{R} 1$ was designed corresponded to the ORF sequence of the transferred gene $\mathrm{CmCYC2c}$, the other pair ClCYC2c-utr-F1/ClCYC2c-utr-R1 was designed according to the $3^{\prime}$ UTR sequence of endogenous $\mathrm{ClCYC2c}$. The ray florets of the three positive lines, showing obvious phenotypic changes, were used to detect the expression levels of all the six ClCYC2 clade genes. The ClActin gene (GenBank accession number JN638568.1) was used as an internal control. All primers were shown in Supplementary Table S1. Both WT plants and transgenic lines expressing the empty vector were used as the negative control plants.

The phenotypes of transgenic lines were observed and recorded during the development process. The numbers of ray and DF were calculated from the same inflorescence using fifteen independent flower heads per line. In addition, the petal ligule lengths of ray florets were measured from 15 inflorescences of each line. The top three fully open inflorescences were sampled from five lateral branches, the second one below each shoot apex, of both positive transgenic lines and control lines. Statistical testing was done with SPSS17.0 (SPSS Inc., Chicago, IL, USA) using one-way ANOVA and Duncan LSD multiple comparisons tests.

\section{RESULTS}

\section{Ontogenesis of Early Flower Primordia of C. morifolium 'Mao xiangyu'}

The capitulum of MXY contains 8-9 whorls of DF and two whorls of peripheral ray florets (Figure 2A). To better understand the morphologic traits of ray and DF of MXY, we normalized the development process into four phases: (I) initiation of floral primordia; (II) differentiation of floral organs; (III) growth of floral organs; and (IV) maturation of inflorescence (data not shown as the capitulum of this stage is big enough to be clearly observed with naked eye). The sizes of the capitula corresponding to each stage were shown in Supplementary Table S2. At stage I, the differentiation of inflorescence primordia was completed and individual flower primordia (arrowed) arose acropetally through the capitulum (Figures 3A,C, SI). The ray and disc floret primordia were indistinguishable undifferentiated bumps at this stage (Figures $\mathbf{3 A}, \mathbf{B}$, SI). At stage IIa, the marginal four whorls of DF began to form petal and stamen primordia (arrowed) while the inner four whorls were still undifferentiated bumps (Figure 3A, SIIa). From stage IIb to IIc, the differentiation of floral organ was continuing, those undifferentiated innermost bumps gradually turned into ringshaped petal primordia (arrowed) with recognizable raised 
stamen primordia (Figure 3A, SIIb). Additionally, the petal lobes of peripheral DF continued to elongate and finally covered the developing stamen and carpel primordia (arrowed, Figure 3A, SIIc). The floral organ differentiation of ray florets seemed to lag behind the outermost DF at stage IIa, which became noticeable at stage IIb when the stamen primordia (st, arrowed) appeared in ray florets (Figure 3B, SIIa and SIIb). In ray florets, the zygomorphy of five petal ligules appeared (arrowed) soon after the petal primordia occurred at stage IIa. Further, the asymmetry became prominent with accelerated expanding of three fused ventral ligules $(\mathrm{vl})$ and ceased growth of two dorsal petals (dp) when the stamen primordia kept on developing (Figure 3B, SIIaSIIc). At stage IId, nearly all ray and DF finished their organ differentiation except for the innermost DF (arrowed, Figure 3A, SIId). The marginal ray florets eventually developed into female with arrested growth of stamens at stage IId, whereas the DF were fully bisexual (Figure 3C, SIId). Furthermore, the hair-like structures (hl) began to emerge on the surface of the peripheral flowers (Figure 3A, SIId). At stage III, the differentiation of floral organs was completed, and all flowers continued to grow and undertook further development to bloom (Figures 3A,B, stage III). In addition, all flowers were attached with those hair-like structures.

\section{Sequence Analysis of the Chrysanthemum CYC-Like Homologs}

To study the role of CYC2 subclade transcription factors in establishment and evolution of the complex flower head of chrysanthemum, six genes encoding these proteins were isolated from young developing inflorescences by an extensive screening of putative homologs from PCR products. These genes were named according to a previously published nomenclature and are as follows: $C m C Y C 2 a, C m C Y C 2 b, C m C Y C 2 c, C m C Y C 2 d$, CmCYC2e, and CmCYC2f (GenBank accession nos. KU595426KU595431). Excluding introns, the length of each gene varied from 774 to $969 \mathrm{bp}$ (258 to 323 inferred amino acids). A conserved motif analysis showed that all these genes contain the conserved TCP and R domain typical for the CYC2/ECE subfamily (Figure $\mathbf{4 B}$ ). The sequence variation among these genes are mainly located within the non-conserved regions (Figure 4C).

We performed phylogenetic analysis on a selected set of TCP class II proteins (between the TCP and R domains) from various plant species to explore the evolutionary relationships and diversification of the $\mathrm{CmCYC2}$ subclade genes. The maximum likelihood analysis placed all the six $\mathrm{CmCYC2}$ genes in CYC2 clade together with all the other listed Asteraceae sequences (Figure 4A). Therefore, our focus here was on the CYC2 subfamily. The most clearly supported orthologous were $\mathrm{CmCYC2a}$ and $\mathrm{HaCYC} 2 a$ along with $\mathrm{GhCYC7}$, as well as the recently duplicated gene pair $\mathrm{CmCYC} 2 b$ and $\mathrm{HaCYC} 2 b$ grouping with $\mathrm{CmCYC2c}$. Although the relationship was not well supported, $\mathrm{CmCYC2f}$ and $\mathrm{CmCYC2e}$ were grouped into one clade, and sister to a Senecio ray floret specific gene SsRAY2. The CmCYC2d gene was clustered with the other Senecio ray floret specific gene SsRAY1. In conclusion, CmCYC2f and $\mathrm{CmCYC2e}$ showed a sister relationship whereas the other four
CmCYC2 genes were sister to the other Asteraceae sequences, suggesting both lineage-specific and shared duplications in Asteraceae.

\section{Expression Patterns of the Chrysanthemum CYC-Like Homologs}

According to the earlier results in Senecio, Gerbera and Helianthus (Broholm et al., 2008; Kim et al., 2008; Fambrini et al., 2011; Tahtiharju et al., 2012), CYC2 subclade genes are involved in regulating the complex inflorescence architecture of Asteraceae. To look into the possible role of the CYC-like genes in chrysanthemum, we investigated the transcription levels of the six $\mathrm{CmCYC2}$ genes in different tissues of F1c (Figure 5A). All the six $\mathrm{CmCYC2}$ genes were mainly expressed in floral reproductive organs, although they were weakly expressed in vegetative organs, in accordance with the results in Gerbera and sunflower (Broholm et al., 2008). In addition, all these genes were found to be predominantly expressed in petal of ray florets and barely expressed in that of DF. $\mathrm{CmCYC} 2 b$ and $\mathrm{CmCYC} 2 c$ in contrast were strongly expressed in petal of middle translike florets, which was not generally true for other $\mathrm{CmCYC2}$ genes. Contrary to the previous studies in other species (Broholm et al., 2008; Tahtiharju et al., 2012), all the CmCYC2 genes were surprisingly relatively highly expressed in the involucrate bracts while slightly expressed in stamens and pistil (including the ovary, styles, and stigma). qRT-PCR reactions were also performed to compare the expression patterns of these genes in flower buds of MXY at both early and late development stages, as shown in Figure 5B. Interestingly, the expression of these genes all peaked at stage IIa and then decreased gradually and maintained at relative low levels, except for $\mathrm{CmCYC2c}$, which was remarkably up-regulated at stage IV (mature flower buds).

To further investigate any flower-type specific role of the CYClike genes, we compared the expression patterns of these genes in two chrysanthemum cultivars and two of their F1 progenies (Figures 2A and 5C). Similar to the previous studies (Broholm et al., 2008; Tahtiharju et al., 2012), all genes were found to be expressed at much higher levels in ray florets than in DF, as shown in Figure 5C, except that $C m C Y C 2 f$ was expressed at more or less comparable levels in all florets. $\mathrm{CmCYC2d}$ and CmCYC2e were expressed at generally similar levels in all of the tested ray florets, and did not display phenotype difference among four types of flower heads. In contrast, the expression of $\mathrm{CmCYC} 2 \mathrm{c}$ was dramatically up-regulated in ray florets of the double-ray flowered heads (GQ and F1a), compared with the expression in the radiate (MXY) and semi-double ray flowered (F1b) heads. Although $\mathrm{CmCYC2a}$ and $\mathrm{CmCYC} 2 b$ exhibited an expression pattern similar to the $\mathrm{CmCYC2c}$, they were expressed at much lower levels.

In short, $\mathrm{CmCYC} 2 \mathrm{c}$ was abundantly expressed at two key stages of petal development in inflorescence primordia, and in petals of both ray and trans-like florets. Moreover, in a series of flower heads with various whorls of ray florets, the transcript levels of $\mathrm{CmCYC2c}$ were significantly up-regulated in ray florets of the double-ray flowered heads. We therefore conclude that $\mathrm{CmCYC2c}$ is a strong candidate as a key regulator of ray floret 
A

B
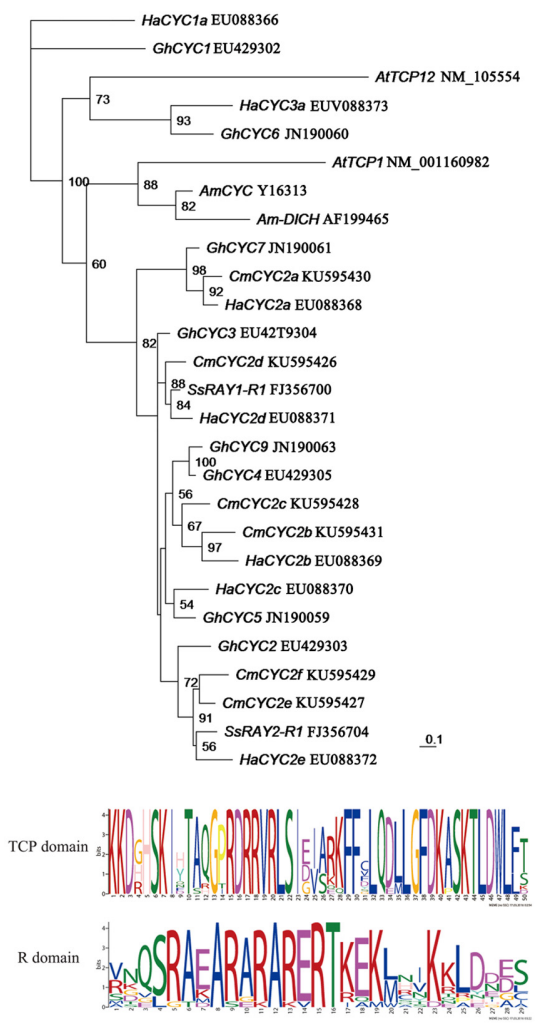

C
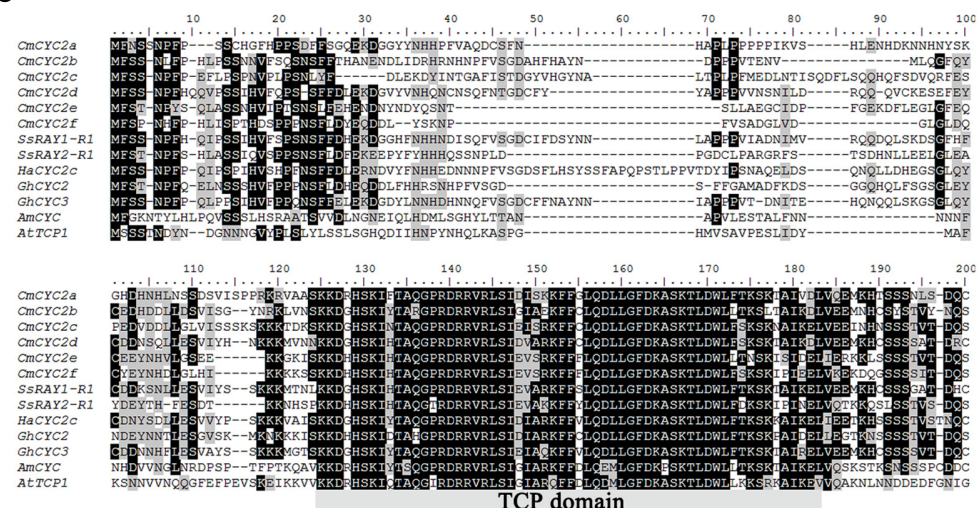

TCP domain

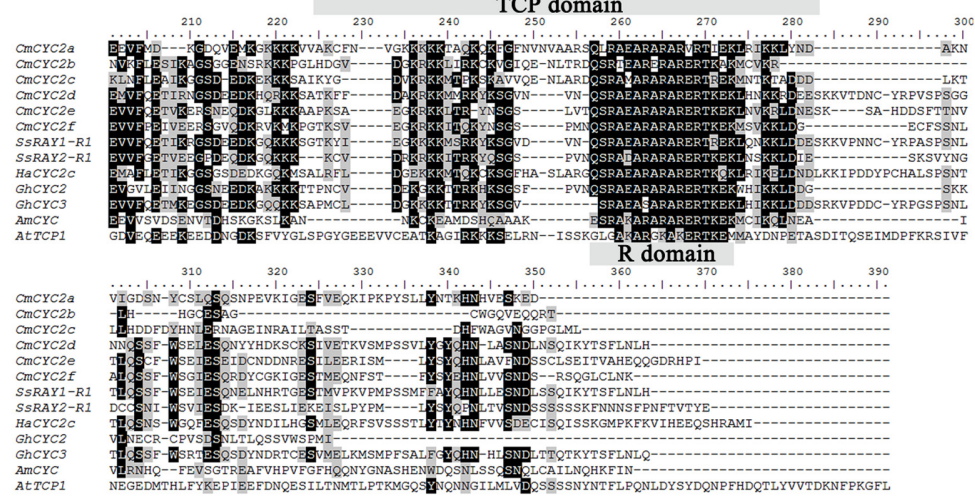

FIGURE 4 | Multiple alignment and phylogenetic analysis of CYC-like proteins from Asteraceae and selected other species. (A) Phylogenetic analysis of selected CYC/TB1-like proteins using the maximum likelihood method. Local bootstrap probabilities are indicated for branches with > 50\% support. The accession numbers of all listed sequences retrieved from GenBank database are shown following gene names. Species abbreviations in gene names are as follows: Am, Antirrhinum majus; At, Arabidopsis thaliana; Gh, Gerbera hybrid; Ha, Helianthus annuus; Cm, Chrysanthemum morifolium; Ss, Senecio squalidus. (B) The two conserved TCP and R domains are analyzed by MEME software. The overall height of the stack indicates the sequence conservation at that position. (C) Alignment of deduced amino acid sequences of $\mathrm{CmCYC2}$ and $\mathrm{CYC}$-like proteins from other organisms. Identical amino acid residues are shaded in black, similar in gray.

identity in chrysanthemum, and hence need a further verification through transgene.

\section{Transgenic Analysis of $\mathrm{CmCYC2c}$ in C. lavandulifolium}

According to our results, $\mathrm{CmCYC2c}$ was predominantly expressed in ray and trans-like florets (if presented) of the double-ray flowered inflorescence. Thus, the ORF of $\mathrm{CmCYC2c}$ was transformed under the CaMV 35s promoter into C. lavandulifolium. Finally, three positive lines showing obvious phenotypic changes were chosen for further analysis. Ectopic expression of $35 \mathrm{~S}:: \mathrm{CmCYC2c}$ retarded the vegetative growth and outgrowth of lateral branches in young plantlets (Figure 6A). However, the growth of these young seedlings restored gradually later on and they produced inflorescence finally (Figure 6B). Further, the capitula appeared larger in the over-expression lines due to increased flower number and elongated petal ligule length of ray florets, compared with the WT plants and transgenic plants with empty vector (Figure 6C). The average length of petal ligule in ray florets was remarkably increased from $4.72 \mathrm{~mm}$ in empty vector to $6.12-7.04 \mathrm{~mm}$ in positive transgenic lines (Figure 6D; Table 1). In addition, the average number of ray florets per inflorescence was significantly enhanced from 13.8 in empty vector to 16-18 in positive transgenic lines (Table 1). Furthermore, we found some trans-like florets in one of the transgenic lines, which were morphologically similar to ray florets but with short petals and abnormal stamens (Figure 6E). In some inflorescences, the growth of ray florets was severally inhibited and resulted in malformation (data not shown). Interestingly, no phenotypic difference was observed in DF among different lines, in accordance with the results in Senecio (Kim et al., 2008; Garcês et al., 2016).

Moreover, the six independent positive transgenic lines that expressed $\mathrm{CmCYC} 2 c$ constitutively (Figure 7A) were further confirmed by qRT-PCR assay (Figure 7B). The expression of $\mathrm{CmCYC} 2 c$ was highly up-regulated in positive lines when compared with that of the endogenous $\mathrm{ClCYC2c}$ gene. Furthermore, given that the $\mathrm{CYC} 2$ clade proteins have been shown to cross-regulate each other to positive regulate gene expression (Tahtiharju et al., 2012; Yang et al., 2012), the effect of $\mathrm{CmCYC2c}$ on the other five $\mathrm{ClCYC2}$ genes was also analyzed by qRT-PCR assay (Figure 7C). The results showed that, besides a significant up-regulation for $\mathrm{ClCYC} 2 c$, the expression of $\mathrm{ClCYC} 2 \mathrm{f}$ was also strongly induced in ray florets of the transgenic lines 
A
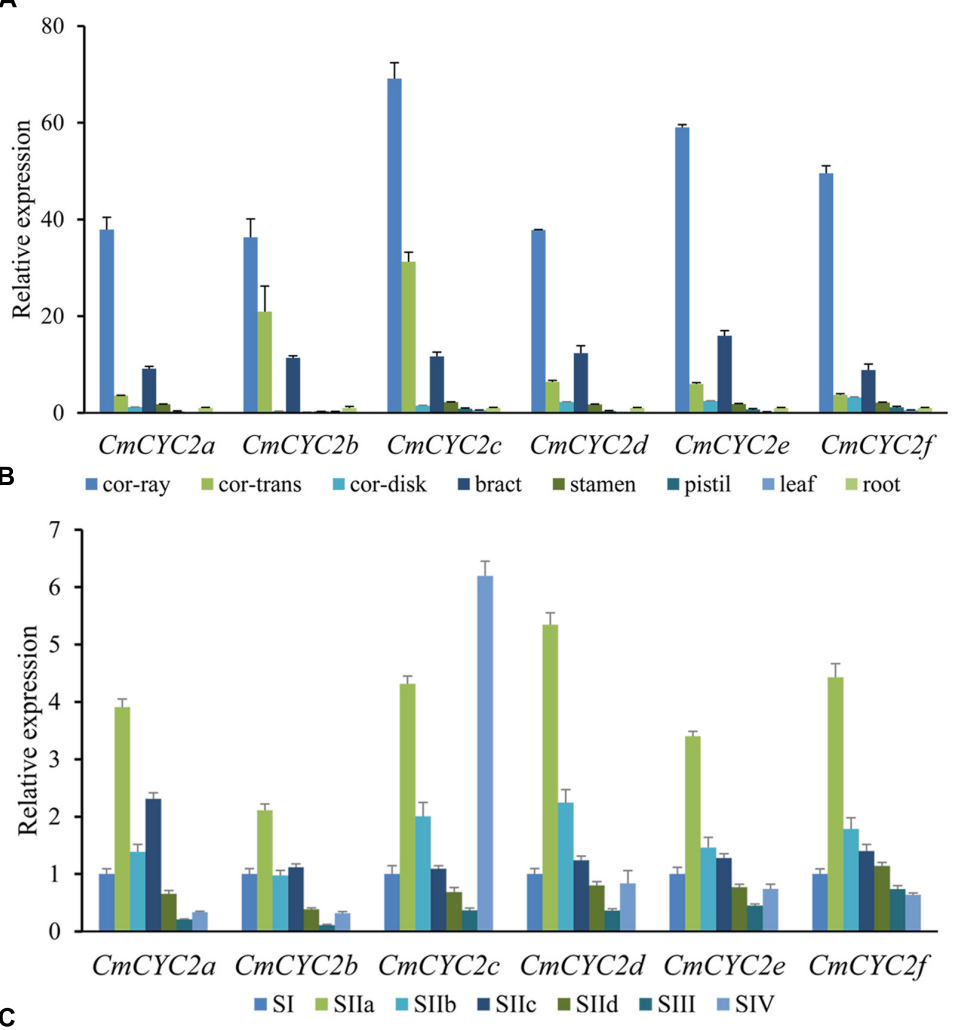

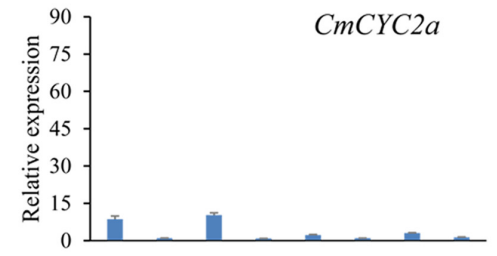

ray disk ray disk ray disk ray disk

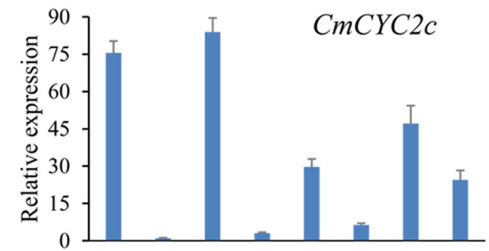

ray disk ray disk ray disk ray disk

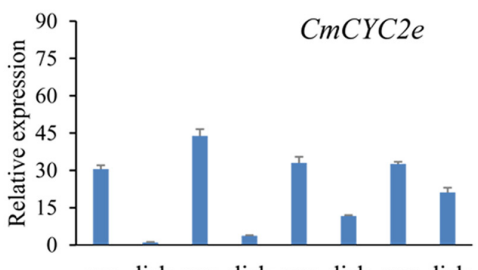

ray disk ray disk ray disk ray disk

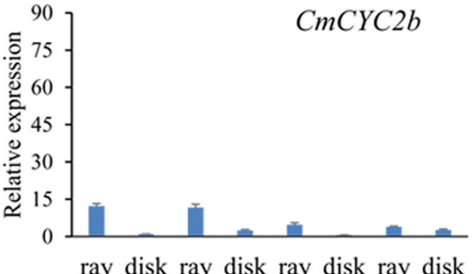

$\mathrm{CmCYC2d}$
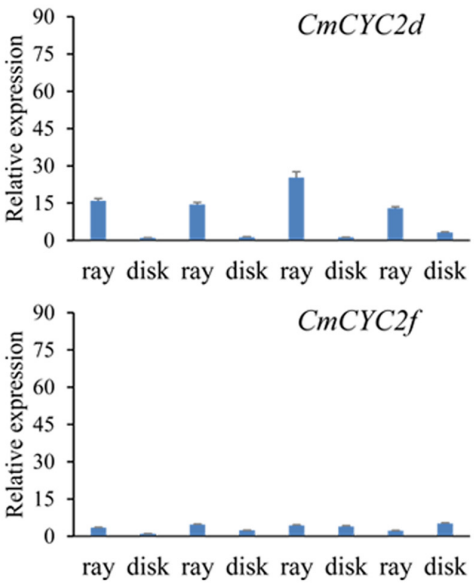

FIGURE 5 | The relative expression levels of the six chrysanthemum CYC2 genes. The columns show average expression levels of three biological replicates for each sample. Expression levels of CmActin were used for normalization. (A) Expression patterns of CmCYC2 genes in different tissues of F1c (a F1 progeny). Tissues analyzed including: corolla of ray florets ( $\mathrm{Cr}$ ), corolla of trans florets (Ct), corolla of disc florets (Cd), involucral bract (Br), pistil (Pi) including ovary, stigma and style, stamen (St), leaf and root. (B) Expression patterns of CmCYC2 genes in flower buds of C. morifolium 'Mao xiangyu' (MXY) during inflorescence development. SI-SIV indicate development stages (stage1-stage IV) of inflorescence. (C) Relative expression analysis of the CmCYC2 subclade genes in two chrysanthemum cultivars and their F1 progenies have intermediate whorls of ray florets. RNA was isolated from the ray florets (ray) and DF (disc) of four types of flower heads. The values were normalized to the CmActin, and were graphically scaled to the disc floret of $\mathrm{GQ}$, with error bars depicting the standard deviation of three biological replicates. 
A

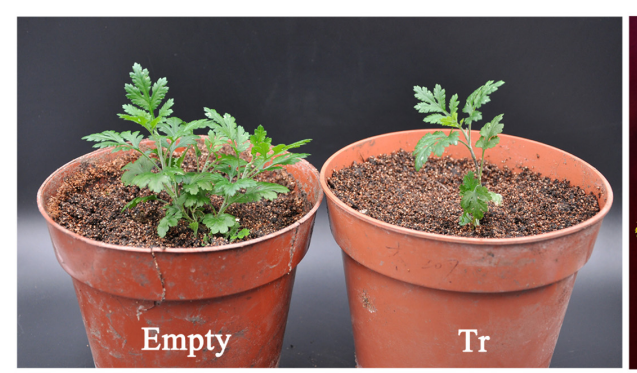

C

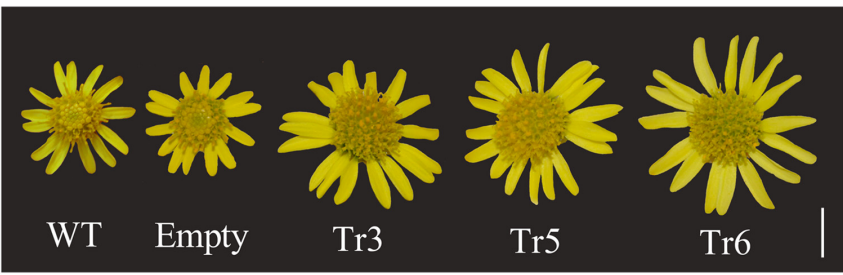

B

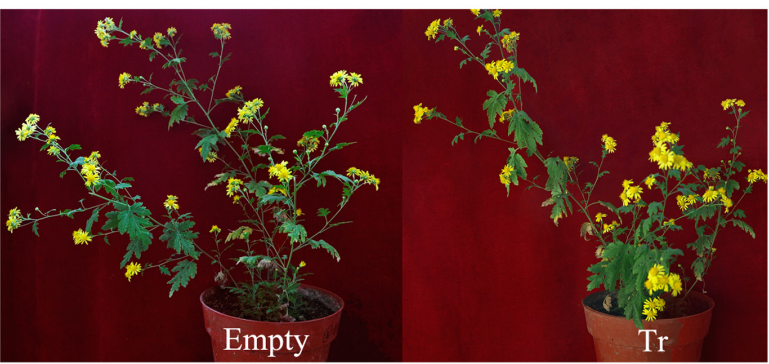

D

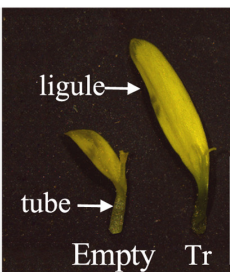

E

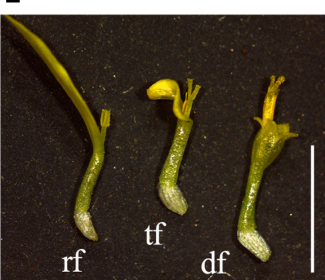

FIGURE 6 | Phenotype of the transgenic plants overexpression $\mathbf{C m C Y C 2 c . ~ ( A ) ~ T h e ~ v e g e t a t i v e ~ g r o w t h ~ o f ~ t h e ~ p o s i t i v e ~ o v e r e x p r e s s i o n ~ l i n e ~ ( T r ) ~ w a s ~ r e t a r d e d ~ a t ~}$ seedling stage compared with that of the line with empty vector (Empty). (B) Phenotype of the blooming plants. (C) The effect of activated CmCYC2c function on the inflorescence. The capitula of positive transgenice lines appear to be larger due to enhanced length of ventral petal ligule in ray florets and increased number of ray florets per head. (D) The ventral petal ligule of ray florets in positive transgenic lines are longer than those in lines with empty vector. (E) Trans-like flowers (tf) are generated between the normal ray ( $(\mathrm{f})$ and disc florets (df) in one transgenic line. (Scale bars: $5 \mathrm{~mm}$ )

when comparing with that in both control ray florets. No obvious difference was found for the expression levels of the other four ClCYC2 genes among these lines.

\section{DISCUSSION}

\section{Duplication and Divergence of Chrysanthemum CYC-Like Genes}

CYC-like genes in Asteraceae have been shown to function as key regulators controlling the differentiation of the specific flower type (Marshall and Abbott, 1984; Broholm et al., 2008; Kim et al., 2008; Fambrini et al., 2014b). Much of the work has focused on the three model species: S. vulgaris (Kim et al., 2008;

TABLE 1 | Ray flower traits affected in the $\mathrm{CmCYC2c}$ transgenic lines in C. lavandulifolium.

\begin{tabular}{lcc}
\hline Genotype & $\begin{array}{c}\text { Ligule length of } \\
\text { ray florets } \mathbf{( m m})\end{array}$ & $\begin{array}{c}\text { Number of ray florets per } \\
\text { capitulum }\end{array}$ \\
\hline Wild-type(WT) & $4.89 \pm 0.49 \mathrm{a}$ & $13.75 \pm 1.37 \mathrm{a}$ \\
35S::Empty vector & $4.72 \pm 0.41 \mathrm{a}$ & $13.8 \pm 1 \mathrm{a}$ \\
35S:: CmCYC2c-TR3 & $6.48 \pm 0.41 \mathrm{c}$ & $16.15 \pm 1.27 \mathrm{~b}$ \\
35S:: CmCYC2C-TR5 & $6.12 \pm 0.27 \mathrm{~b}$ & $18.1 \pm 1.55 \mathrm{c}$ \\
35S:: CmCYC2C-TR6 & $7.04 \pm 0.71 \mathrm{~d}$ & $16.2 \pm 1.36 \mathrm{~b}$ \\
\hline
\end{tabular}

Mean values $\pm S D$ are analyzed with a one-way ANOVA. Different letters adjacent to each value in the same column indicate significant differences at $P<0.05$ by Duncan LSD test. The length of the petal ligule of ray florets and the number of ray florets per capitulum were documented from three independent transgenic lines in comparison with WT plants and transgenic plants with empty vector.
Garcês et al., 2016), H. annuus (Chapman et al., 2008, 2012) and G. hybrid (Broholm et al., 2008; Juntheikki-Palovaara et al., 2014). Surprisingly, few reports have been published on the function of these genes in the chrysanthemum, which is characterized by the extreme diversity of floral morphology. It has been previously reported that the CYC2 subclade has undergone the greatest number of gene duplications within the Asteraceae, such as five CYC-like genes in H. annuus and six in Gerbera (Broholm et al., 2008). No more copies have previously been reported in any other plant lineages studied thus far. Correspondingly, six $\mathrm{CmCYC2}$ genes were obtained in chrysanthemum with highly conserved TCP and R domains. Gene and whole-genome duplications in plants are known to increase genomic complexity and have been suggested to produce new genes that allow the evolution of functional novelty (Jiao et al., 2011). The duplication of CYC2 subclade in Asteraceae seems to support the idea that members of this subclade may have novel and important functions contributing to the complex inflorescence structure in Asteraceae. Intriguingly, the strong gene candidates in specifying flower zygomorphy in Asteraceae were grouped into different clades according to the phylogenetic trees reported previously by Chapman et al. (2012) and Tahtiharju et al. (2012) and the current study. For example the $\mathrm{HaCYC} 2 \mathrm{c}$ in sunflower (Chapman et al., 2012), the GhCYC2 in Gerbera (Tahtiharju et al., 2012), the SsRAY1 and SsRAY2 in Senecio (Kim et al., 2008), and the $\mathrm{CmCYC2c}$ in chrysanthemum were placed into five different groups, rather than showing sister relationships. These results indicate that paralogs of this gene family have been independently recruited to regulate zygomorphy in different species within the Asteraceae. Moreover, similar to the reports in sunflower and C. lavandulifolium (Tahtiharju et al., 2012; Ren and Guo, 


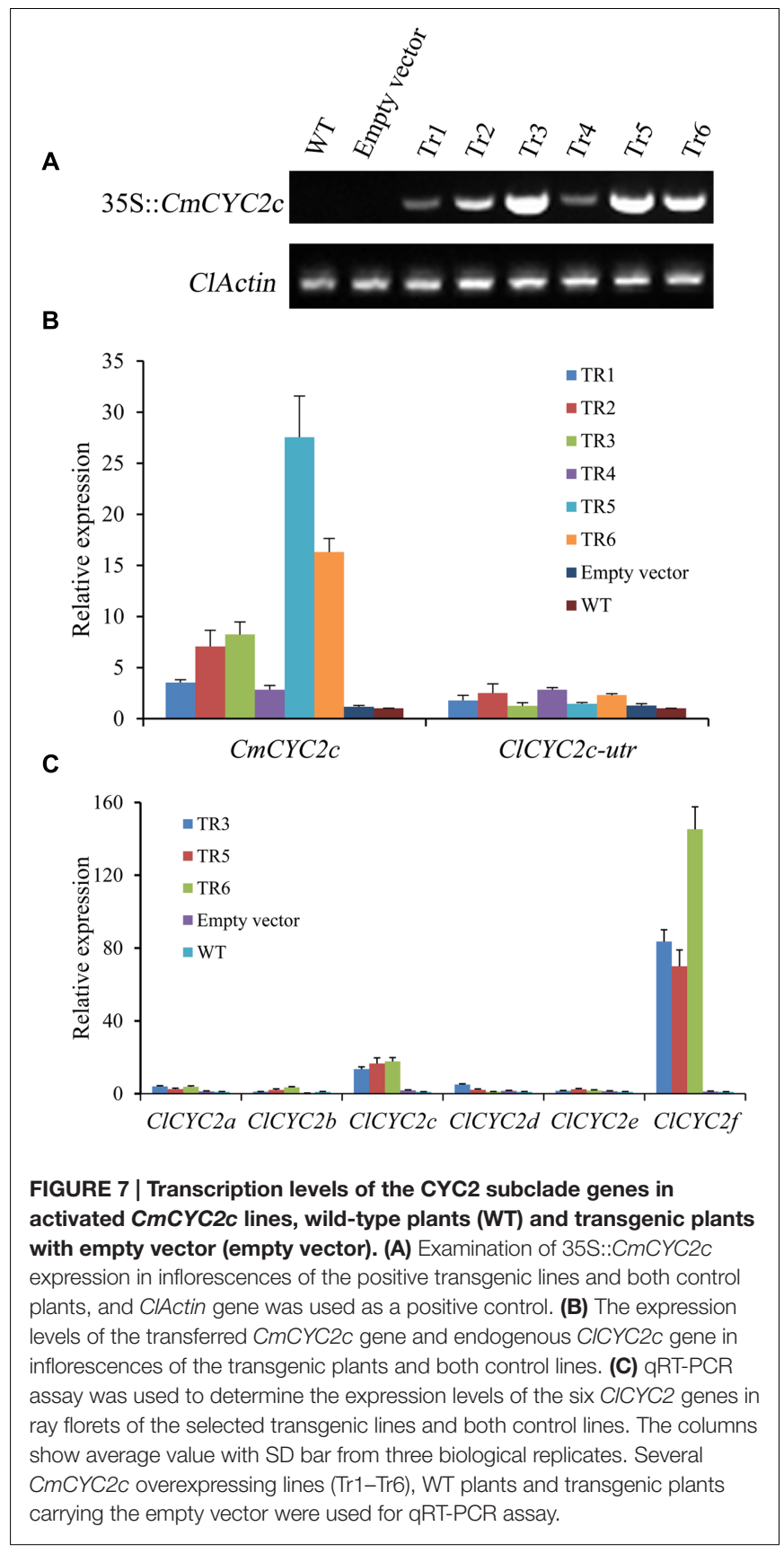

2015), ray florets could be easily distinguished from disc ones in chrysanthemum at early stage of flower primordia development. The reason is that the asymmetric growth of the five petals in ray florets occurred soon after the petal primordia emerged. In Gerbera, however, the ray and disc petal primordia do not show difference in growth until late developing stages (Laitinen et al., 2006; Tahtiharju et al., 2012). Thus, the morphological divergence within Asteraceae in this respect is also consistent with the organismal phylogeny. As G. hybrida (Mutisieae) is a phylogenetically more distant specie when compared with the other three species (Bremer and Anderberg, 1994; Panero and Funk, 2008). This explanation can be further supported by the phylogenetic analysis that all the $\mathrm{CmCYC2}$ genes were sister to the corresponding CYC2 members of sunflower and Senecio, whereas they were not clustered with most Gerbera CYC2 genes.

\section{Expression Analysis Indicates both Early and Late Functions for $\mathrm{CmCYC2c}$ during Ray Floret Development}

Typically, the expression of CYC2 subclade genes is restricted to the dorsal regions of the flower, for example, in A. majus (Luo et al., 1999) and Veronica montana (Preston et al., 2009). In Asteraceae, however, the expression of CYC-like genes is excluded from the dorsal petals and has shifted to the ventral part of ray florets (Chapman et al., 2008; Kim et al., 2008; Tahtiharju et al., 2012; Juntheikki-Palovaara et al., 2014). For example, RAY1, RAY2, and RAY3 in Senecio, proved to control ray versus disc floret identity, are expressed only in ray florets (Kim et al., 2008; Garcês et al., 2016). Here in chrysanthemum, the $\mathrm{CmCYC} 2$ genes were also predominantly expressed in petals of ray florets, consistent with what has been reported previously in Asteraceae (Chapman et al., 2008; Tahtiharju et al., 2012). The appearance of trans-like florets in the F1 progeny (F1c) suggests it is a transition type between ray and DF. In addition to petals of ray florets, $\mathrm{CmCYC} 2 c$ and $\mathrm{CmCYC} 2 b$ were also abundantly expressed in petals of trans-like florets, suggesting their possible role in promoting the growth of ventral petal ligule. This fits well with our data in transgenic lines that over-expression of $\mathrm{CmCYC} 2 c$ leads to elongated ventral ligule length in ray florets. Similar results have been reported for GhCYC4, which also shows ray/TF specific expression, and function in enhancing the growth of petal ligules (Juntheikki-Palovaara et al., 2014). Detailed expression analysis in Gerbera show that GhCYC2 is exclusively expressed in the ventral domain, and the expression

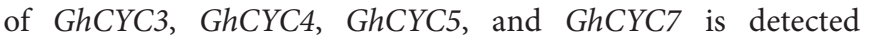
both in the fused ventral ligule and two rudimentary dorsal petals.

The transcript levels of the six $\mathrm{CmCYC2}$ genes were analyzed in two chrysanthemum cultivars and their F1 progenies with intermediate whorls of ray florets. All these genes were predominantly expressed in ray florets, and most of them showed no or some expression variation across genotypes. However, the expression of $\mathrm{CmCYC} 2 \mathrm{c}$ was significantly upregulated in the double-ray flowered heads, indicating it is a strong candidate as a regulator of ray floret identity. It is supported by the fact that the $35 \mathrm{~s}:: C m C Y C 2 c$ transgenic lines generate more ray florets per capitulum in comparison with that in the control lines. Despite well-known for the abundant flower shape, nearly all wild original crossing parents of modern chrysanthemum are characterized by multiple whorls of DF surround by a single whorl of peripheral ray florets. Of these wild original germplasm, C. lavandulifolium is one typical and vital diploid specie. The regulatory mechanism controlling the growth of ray florets in chrysanthemum is still not clear yet. Therefore, it is interesting and meaningful that the number and morphology of the ray florets of C. lavandulifolium are 
changed by ectopic activation of $\mathrm{CmCYC} 2 \mathrm{c}$. According to the reports by Tahtiharju et al. (2012), HaCYC2c and GhCYC3 are indicated to be strong candidates in controlling of ray floret identity owing to their specific expression in ray flowers. In sunflower, the expression of $\mathrm{HaCYC} 2 b$ and $\mathrm{HaCYC} 2 e$ are generally similar across different genotypes, whereas increased $\mathrm{HaCYC} 2 \mathrm{c}$ expression is detected across the inflorescence of double-flowered ( $d b l$ ) mutant (with only ray florets) (Chapman et al., 2008, 2012). Furthermore, overexpression of $\mathrm{HaCYC} 2 \mathrm{c}$ due to an insertion upstream of the start codon generates $d b l$ mutant, providing functional evidence for its role in ray formation (Chapman et al., 2012). In addition, GhCYC3 in Gerbera is highly up-regulated in the centermost ray floret primordia of the crested cultivar, which can convert DF into ray-like ones by promoting petal ligule growth in transgenic lines (Broholm et al., 2008).

The CmCYC2 subclade genes also share strikingly similar expression patterns during the early inflorescence developing stages (from SI to SIII). All these genes were highly expressed at stage IIa and then decreased gradually. However, $\mathrm{CmCYC} 2 \mathrm{c}$ was also highly expressed at stage IV when compared with the other five genes. Since the petal of ray florets begin to differentiate at stage IIa and further expand at stage IV, we speculate that $\mathrm{CmCYC} 2 \mathrm{c}$ may function in regulating petal development at both early and late stages. Similar functions have been reported previously for $C Y C$ and $G h C Y C 3$, which are involved in modifying petal growth owing to cell expansion or proliferation at different stages of development (Luo et al., 1996; JuntheikkiPalovaara et al., 2014). However, further studies are required to elucidate the expression patterns of the $C m C Y C 2$ genes at the organ and tissue level using, for example, RNA in situ hybridization.

\section{Overexpression of $\mathrm{CmCYC2c}$ in C. lavandulifolium Promotes the Petal Growth of Ray Florets}

An increasing number of evidence demonstrate that CYC-like genes participate in controlling flower symmetry and typically the growth of the dorsal parts of a flower as shown, for example, in A. majus (Luo et al., 1996, 1999) and I. amara (Busch and Zachgo, 2007). In Antirrhinum, for example, CYC and $\mathrm{DICH}$ regulate flower symmetry by promoting dorsal petal growth but repressing dorsalmost stamen development (Luo et al., 1996, 1999). Similarly, in Mohavea confertiflora, the expression of $\mathrm{McCYC}$ and $\mathrm{McDICH}$ in both dorsal and lateral regions is correlated with the abortion of both dorsal and lateral stamens and the increase of dorsal petal sizes (Hileman and Baum, 2003). In addition to controlling symmetry in a single flower, the TCP domain regulatory proteins in Asteraceae have developed a novel role in regulation of floral identity within the capitulum (Broholm et al., 2008; Fambrini et al., 2014b; Juntheikki-Palovaara et al., 2014; Garcês et al., 2016). Abundant expression data have shown that these members of CYC2 subclade in Asteraceae play a pivotal role in promoting or repressing the growth of ventral petal ligule, and sometimes affecting stamen development, hence change the symmetry of corresponding flowers (Broholm et al., 2008; Fambrini et al., 2014b; Juntheikki-Palovaara et al., 2014; Garcês et al., 2016).

Our transgenic data show that overexpression of $\mathrm{CmCYC} 2 \mathrm{c}$ in C. lavandulifolium leads to significant elongation in ventral ligule length of ray florets. Based on the transgenic phenotype, the high expression of $\mathrm{CmCYC} 2 \mathrm{c}$ in the petals of ray and translike florets appears to be crucial for the ventral petal ligule growth. This is in consistent with the results in Gerbera, in which overexpression of GhCYC3 and GhCYC4 enhances petal ligule growth in trans flowers (Juntheikki-Palovaara et al., 2014). Conversely, ectopic activation of GhCYC2, GhCYC3, and RAY1 can reduce ventral petal growth of corresponding ray florets (Kim et al., 2008; Juntheikki-Palovaara et al., 2014). Moreover, in sunflower and gerbera, overexpression of $\mathrm{HaCYC} 2 \mathrm{c}, \mathrm{GhCYC2}$, and GhCYC3 can cause their DF to obtain some characteristics of ray florets, while no effect is observed on the DF of RAY1, RAY2, and RAY3 transgenic Senecio lines (Chapman et al., 2012; Juntheikki-Palovaara et al., 2014; Garcês et al., 2016). In this study, although one transgenic line generated some trans-like flowers, no difference was observed in DF between transgenic lines and control lines. Therefore, it appears that the CYC2 subclade proteins in Asteraceae have experienced functional diversification. Similar functional deviations have also been reported in regulation of stamen development (Juntheikki-Palovaara et al., 2014; Garcês et al., 2016). Besides promoting ventral petal length of ray florets, ectopic activation of $\mathrm{CmCYC} 2 \mathrm{c}$ also statistically increased the number of ray florets per capitulum. Several studies have shown that the showy marginal flowers in Asteraceae increases the attractiveness of the inflorescence to pollinators, thereby increasing genetic diversity and fitness (Marshall and Abbott, 1984; Bremer and Anderberg, 1994; Andersson, 2008). Contrary to the single whorl of ray florets with changeless shape in the wild original species, the modern chrysanthemum cultivars are characterized by the various whorls of ray florets and the diversity of petal shape. Taking together the high expression levels of $\mathrm{CmCYC} 2 \mathrm{c}$ in ray florets of the double-ray flowered chrysanthemum and the transgenic phenotypes in C. lavandulifolium, we can postulate that the $\mathrm{CmCYC} 2 \mathrm{c}$ could be a vital regulator in evolution of modern chrysanthemum.

CYC2 clade proteins have been shown to influence vegetative and reproductive growth to produce morphological novelties in plants (Cubas et al., 1999b; Costa et al., 2005; Zhong and Kellogg, 2015). In this study, over expression of $\mathrm{CmCYC} 2 c$ in C. lavandulifolium resulted in retarded vegetative growth and reduced branching of young plantlets, which were restored gradually later, fortunately. Similar effect has been reported on many other TCP transcription factors. For example in Gerbera, constitutive expression of GhCYC2 causes severe growth defects and difficulties in regeneration of transgenic shoots (Broholm et al., 2008). Similarly, ectopic activation of CYC-like genes in Arabidopsis leads to reduction in vegetative growth (Costa et al., 2005; Yang et al., 2012; Juntheikki-Palovaara et al., 2014). Thus, at least in vegetative organs, their function is conserved. However, negative regulation of bud formation or branching outgrowth is mainly documented for some other genes in CYC/TB1 group, such as TB1 in maize (Doebley et al., 1995; 
Hubbard et al., 2002), OsTB1 in rice (Takeda et al., 2003) and BRC1 in Arabidopsis (Aguilar-Martínez et al., 2007). Conversely, heterologous expression of $C Y C 1 C$ in Arabidopsis promotes the outgrowth of lateral branches (Yang et al., 2012). Therefore, it seems like that ECE clade genes have undergone functional divergence in this content.

\section{The Expression of $\mathrm{CmCYC2f}$ Is Strongly Up-regulated in $\mathrm{CmCYC2c}$ Overexpressing Lines}

Intriguingly, overexpression of $\mathrm{CmCYC} 2 \mathrm{c}$ can strongly induce the expression of $\mathrm{CmCYC} 2 f$ in all transgenic lines. According to the reports in Primulina, CYC1C and CYC1D can positively autoregulate themselves and cross-regulate each other to form auto-regulatory loops, which may trigger threshold-dependent genetic witches (Yang et al., 2012). In addition, Tahtiharju et al. (2012) shows that Gerbera CYC2 clade proteins have the ability of interacting in yeast two-hybrid assays, and the co-regulators may target different downstream genes. Therefore, we postulate that $C m C Y C 2 f$ may work as a putative downstream target of $\mathrm{CmCYC} 2 c$ to induce the differentiation of specific flower in chrysanthemum. Functional analysis of $C m C Y C 2 f$ is currently underway.

\section{CONCLUSION}

We show for the first time that CYC-like genes in chrysanthemum have undergone gene duplication and functional divergence, a condition that appears to be connected with the increased inflorescence complexity. Transcription analysis demonstrates that most of the chrysanthemum CYC-like genes are mainly expressed in ray florets and therefore may be functionally redundant. Of these $\mathrm{CYC} 2$ subclade genes, $\mathrm{CmCYC} 2 c$ can

\section{REFERENCES}

Aguilar-Martínez, J. A., Poza-Carrión, C., and Cubas, P. (2007). Arabidopsis BRANCHED1 acts as an integrator of branching signals within axillary buds. Plant Cell 19, 458-472. doi: 10.1105/tpc.106.048934

Almeida, J., Rocheta, M., and Galego, L. (1997). Genetic control of flower shape in Antirrhinum majus. Development 124, 1387-1392.

Andersson, S. (2008). Pollinator and nonpollinator selection on ray morphology in Leucanthemum vulgare (oxeye daisy, Asteraceae). Am. J. Bot. 95, 1072-1078. doi: $10.3732 / a j b .0800087$

Bradley, D., Carpenter, R., Sommer, J., Hartley, N., and Coen, E. S. (1993). Complementary floral homeotic phenotypes result from opposite orientations of a transposon at the plena locus of Antirrhinum. Cell 72, 85-95. doi: 10.1016/0092-8674(93)90052-R

Bremer, K., and Anderberg, A. A. (1994). Asteraceae: Cladistics \& Classification. Portland, OR: Timber Press.

Broholm, S. K., Tahtiharju, S., Laitinen, R. A., Albert, V. A., Teeri, T. H., and Elomaa, P. (2008). A TCP domain transcription factor controls flower type specification along the radial axis of the Gerbera (Asteraceae) inflorescence. Proc. Natl. Acad. Sci. U.S.A. 105, 9117-9122. doi: 10.1073/pnas.080135 9105

Busch, A., and Zachgo, S. (2007). Control of corolla monosymmetry in the Brassicaceae Iberis amara. Proc. Natl. Acad. Sci. U.S.A. 104, 16714-16719. doi: 10.1073/pnas.0705338104 easily be identified as a vital gene in regulating ray floret identity. Moreover, overexpression of $\mathrm{CmCYC} 2 \mathrm{c}$ is capable of regulating the growth of ray florets, whereas it is not sufficient for completely changing the floral shape. Therefore, except for $\mathrm{CmCYC} 2 c$, whether the other transcription factors, such as the other $\mathrm{CmCYC2}$ members, $M Y B$-domain transcription factors RADIALIS (Corley et al., 2005; Garcês et al., 2016) and DIVARICATA (Almeida et al., 1997), are involved in regulating ray floret identity in chrysanthemum awaits further study.

\section{AUTHOR CONTRIBUTIONS}

DH and QZ conceived and designed the experiments. XL and MS prepared the plant materials. DH and TZ performed experiments. JW, HP, and TC contributed reagents and analysis tools. $\mathrm{DH}$ wrote the paper.

\section{ACKNOWLEDGMENTS}

This work was supported by the 12th Five Years Key Programs for Science and Technology Development of China (2013BAD01B07, 2012BAD01B07), Special Fund for Beijing Common Construction Project.

\section{SUPPLEMENTARY MATERIAL}

The Supplementary Material for this article can be found online at: http://journal.frontiersin.org/article/10.3389/fpls.2016.01633/ full\#supplementary-material

FIGURE S1 | Inflorescence developmental stages 1, 3, 4, 6, 7, 8, and 9 of C. morifolium 'Mao xiangyu' (MXY). Stage 1 is corresponding to the developing phase of stage IV.

Chapman, M. A., and Abbott, R. J. (2010). Introgression of fitness genes across a ploidy barrier. New Phytol. 186, 63-71. doi: 10.1111/j.1469-8137.2009.03091.x

Chapman, M. A., Leebens-Mack, J. H., and Burke, J. M. (2008). Positive selection and expression divergence following gene duplication in the sunflower CYCLOIDEA gene family. Mol. Biol. Evol. 25, 1260-1273. doi: 10.1093/molbev/msn001

Chapman, M. A., Tang, S., Draeger, D., Nambeesan, S., Shaffer, H., Barb, J. G., et al. (2012). Genetic analysis of floral symmetry in Van Gogh's sunflowers reveals independent recruitment of CYCLOIDEA genes in the Asteraceae. PLoS Genet. 8:e1002628. doi: 10.1371/journal.pgen.1002628

Chen, G., Hackett, R., Walker, D., Taylor, A., Lin, Z., and Grierson, D. (2004). Identification of a specific isoform of tomato lipoxygenase (TomloxC) involved in the generation of fatty acid-derived flavor compounds. Plant Physiol. 136, 2641-2651. doi: 10.1104/pp.104.041608

Citerne, H. L., Pennington, R. T., and Cronk, Q. C. (2006). An apparent reversal in floral symmetry in the legume Cadia is a homeotic transformation. Proc. Natl. Acad. Sci. U.S.A. 103, 12017-12020. doi: 10.1073/pnas.0600986103

Corley, S. B., Carpenter, R., Copsey, L., and Coen, E. (2005). Floral asymmetry involves an interplay between TCP and MYB transcription factors in Antirrhinum. Proc. Natl. Acad. Sci. U.S.A. 102, 5068-5073. doi: 10.1073/pnas.0501340102

Costa, M. M., Fox, S., Hanna, A. I., Baxter, C., and Coen, E. (2005). Evolution of regulatory interactions controlling floral asymmetry. Development 132, 5093-5101. doi: 10.1242/dev.02085 
Cubas, P., Coen, E., and Zapater, J. M. (2001). Ancient asymmetries in the evolution of flowers. Curr. Biol. 11, 1050-1052. doi: 10.1016/S0960-9822(01) 00295-0

Cubas, P., Lauter, N., Doebley, J., and Coen, E. (1999a). The TCP domain: a motif found in proteins regulating plant growth and development. Plant J. 18, 215-222. doi: 10.1046/j.1365-313X.1999.00444.x

Cubas, P., Vincent, C., and Coen, E. (1999b). An epigenetic mutation responsible for natural variation in floral symmetry. Nature 401, 157-161. doi: 10.1038/43657

Doebley, J., Stec, A., and Gustus, C. (1995). teosinte branched1 and the origin of maize: evidence for epistasis and the evolution of dominance. Genetics 141, $333-346$.

Endress, P. K. (1999). Symmetry in flowers: diversity and evolution. Int. J. Plant Sci. 160, S3-S23. doi: 10.1086/314211

Fambrini, M., Basile, A., Salvini, M., and Pugliesi, C. (2014a). Excisions of a defective transposable CACTA element (Tetu1) generate new alleles of a CYCLOIDEA-like gene of Helianthus annuus. Gene 549, 198-207. doi: 10.1016/j.gene.2014.07.018

Fambrini, M., Salvini, M., Basile, A., and Pugliesi, C. (2014b). Transposondependent induction of Vincent van Gogh's sunflowers: exceptions revealed. Genesis 52, 315-327. doi: 10.1002/dvg.22743

Fambrini, M., Salvini, M., and Pugliesi, C. (2011). A transposon-mediate inactivation of a CYCLOIDEA-like gene originates polysymmetric and androgynous ray flowers in Helianthus annuus. Genetica 139, 1521-1529. doi: 10.1007/s10709-012-9652-y

Feng, X., Zhao, Z., Tian, Z., Xu, S., Luo, Y., Cai, Z., et al. (2006). Control of petal shape and floral zygomorphy in Lotus japonicus. Proc. Natl. Acad. Sci. U.S.A. 103, 4970-4975. doi: 10.1073/pnas.0600681103

Funk, V. A. (2009). Systematics, Evolution, and Biogeography of Compositae. Vienna: International Association for Plant Taxonomy.

Garcês, H. M. P., Spencer, V. M. R., and Kim, M. (2016). Control of floret symmetry by RAY3, SvDIV1B and SvRAD in the capitulum of Senecio vulgaris. Plant Physiol. 171, 2055-2068. doi: 10.1104/pp.16. 00395

Gillies, A. C. M., Cubas, P., Coen, E. S., and Abbott, R. J. (2002). "Making rays in the Asteraceae: genetics and evolution of radiate versus discoid ?ower heads," in Developmental Genetics and Plant Evolution, eds Q. C. B. Cronk, R. M. Bateman, and J. A. Hawkins (London: Taylor \& Francis).

Guindon, S., Dufayard, J.-F., Lefort, V., Anisimova, M., Hordijk, W., and Gascuel, O. (2010). New algorithms and methods to estimate maximumlikelihood phylogenies: assessing the performance of PhyML 3.0. Syst. Biol. 59, 307-321. doi: 10.1093/sysbio/syq010

Hileman, L. C. (2014). Trends in flower symmetry evolution revealed through phylogenetic and developmental genetic advances. Philos. Trans. R. Soc. Lond. B Biol. Sci. 369:20130348. doi: 10.1098/rstb.2013. 0348

Hileman, L. C., and Baum, D. A. (2003). Why do paralogs persist? Molecular evolution of CYCLOIDEA and related floral symmetry genes in Antirrhineae (Veronicaceae). Mol. Biol. Evol. 20, 591-600. doi: 10.1093/molbev/ msg063

Howarth, D. G., and Donoghue, M. J. (2006). Phylogenetic analysis of the "ECE" (CYC/TB1) clade reveals duplications predating the core eudicots. Proc. Natl. Acad. Sci. U.S.A. 103, 9101-9106. doi: 10.1073/pnas.06028 27103

Hubbard, L., McSteen, P., Doebley, J., and Hake, S. (2002). Expression patterns and mutant phenotype of teosinte branched 1 correlate with growth suppression in maize and teosinte. Genetics 162, 1927-1935.

Jiao, Y., Wickett, N. J., Ayyampalayam, S., Chanderbali, A. S., Landherr, L., Ralph, P. E., et al. (2011). Ancestral polyploidy in seed plants and angiosperms. Nature 473, 97-100. doi: 10.1038/nature09916

Juntheikki-Palovaara, I., Tähtiharju, S., Lan, T., Broholm, S. K., Rijpkema, A. S., Ruonala, R., et al. (2014). Functional diversification of duplicated CYC2 clade genes in regulation of inflorescence development in Gerbera hybrida (Asteraceae). Plant J. 79, 783-796. doi: 10.1111/tpj.12583

Kim, M., Cui, M. L., Cubas, P., Gillies, A., Lee, K., Chapman, M. A., et al. (2008). Regulatory genes control a key morphological and ecological trait transferred between species. Science 322, 1116-1119. doi: 10.1126/science. 1164371
Laitinen, R. A., Broholm, S., Albert, V. A., Teeri, T. H., and Elomaa, P. (2006). Patterns of MADS-box gene expression mark flower-type development in Gerbera hybrida (Asteraceae). BMC Plant Biol. 6:11. doi: 10.1186/1471-22296-11

Laitinen, R. A., Pollanen, E., Teeri, T. H., Elomaa, P., and Kotilainen, M. (2007). Transcriptional analysis of petal organogenesis in Gerbera hybrida. Planta 226, 347-360. doi: 10.1007/s00425-007-0486-2

Larkin, M. A., Blackshields, G., Brown, N. P., Chenna, R., McGettigan, P. A., McWilliam, H., et al. (2007). Clustal W and Clustal X version 2.0. Bioinformatics 23, 2947-2948. doi: 10.1093/bioinformatics/btm404

Liu, P.-L., Wan, Q., Guo, Y.-P., Yang, J., and Rao, G.-Y. (2012). Phylogeny of the genus Chrysanthemum L.: evidence from single-copy nuclear gene and chloroplast DNA sequences. PLoS ONE 7:e48970. doi: 10.1371/journal. pone. 0048970

Luo, D., Carpenter, R., Copsey, L., Vincent, C., Clark, J., and Coen, E. (1999). Control of organ asymmetry in flowers of Antirrhinum. Cell 99, 367-376. doi: 10.1016/S0092-8674(00)81523-8

Luo, D., Carpenter, R., Vincent, C., Copsey, L., and Coen, E. (1996). Origin of floral asymmetry in Antirrhinum. Nature 383, 794-799. doi: 10.1038/ $383794 \mathrm{a} 0$

Marshall, D. F., and Abbott, R. J. (1984). Polymorphism for outcrossing frequency at the ray floret locus in Senecio vulgaris L. II: confirmation. Heredity 52, 331-336. doi: 10.1038/hdy. 1984.40

Panero, J. L., and Funk, V. A. (2008). The value of sampling anomalous taxa in phylogenetic studies: major clades of the Asteraceae revealed. Mol. Phylogenet. Evol. 47, 757-782. doi: 10.1016/j.ympev.2008.02.011

Preston, J. C., Kost, M. A., and Hileman, L. C. (2009). Conservation and diversification of the symmetry developmental program among close relatives of snapdragon with divergent floral morphologies. New Phytol. 182, 751-762. doi: 10.1111/j.1469-8137.2009.02794.x

Qi, S., Yang, L., Wen, X., Hong, Y., Song, X., Zhang, M., et al. (2016). Reference gene selection for RT-qPCR analysis of flower development in Chrysanthemum morifolium and Chrysanthemum lavandulifolium. Front. Plant Sci. 7:287. doi: $10.3389 /$ fpls.2016.00287

Ren, J.-B., and Guo, Y.-P. (2015). Behind the diversity: ontogenies of radiate, disciform, and discoid capitula of Chrysanthemum and its allies. J. Syst. Evol. 53, 520-528. doi: 10.1111/jse.12154

Sargent, R. D. (2004). Floral symmetry affects speciation rates in angiosperms. Proc. R. Soc. Lond. Biol. Sci. 271, 603-608. doi: 10.1098/rspb.2003. 2644

Sun, M., and Ganders, F. R. (1990). Outcrossing rates and allozyme variation in rayed and rayless morphs of Bidens pilosa. Heredity 64, 139-143. doi: 10.1038/hdy.1990.18

Tahtiharju, S., Rijpkema, A. S., Vetterli, A., Albert, V. A., Teeri, T. H., and Elomaa, P. (2012). Evolution and diversification of the CYC/TB1 gene family in Asteraceae-a comparative study in Gerbera (Mutisieae) and sunflower (Heliantheae). Mol. Biol. Evol. 29, 1155-1166. doi: 10.1093/molbev/ msr283

Takeda, T., Suwa, Y., Suzuki, M., Kitano, H., Ueguchi-Tanaka, M., Ashikari, M., et al. (2003). The OsTB1 gene negatively regulates lateral branching in rice. Plant J. 33, 513-520. doi: 10.1046/j.1365-313X.2003.01648.x

Teixeira da Silva, J. A. (2003). Chrysanthemum: advances in tissue culture, cryopreservation, postharvest technology, genetics and transgenic biotechnology. Biotechnol. Adv. 21, 715-766. doi: 10.1016/S0734-9750(03) 00117-4

Teixeira da Silva, J. A., Shinoyama, H., Aida, R., Matsushita, Y., Raj, S. K., and Chen, F. (2013). Chrysanthemum biotechnology: quo vadis? Crit. Rev. Plant Sci. 32, 21-52. doi: 10.1080/07352689.2012.696461

Yang, X., Pang, H. B., Liu, B. L., Qiu, Z. J., Gao, Q., Wei, L., et al. (2012). Evolution of double positive autoregulatory feedback loops in CYCLOIDEA2 clade genes is associated with the origin of floral zygomorphy. Plant Cell 24, 1834-1847. doi: 10.1105/tpc.112.099457

Yang, X., Zhao, X. G., Li, C. Q., Liu, J., Qiu, Z. J., Dong, Y., et al. (2015). Distinct regulatory changes underlying differential expression of TEOSINTE BRANCHED1-CYCLOIDEA-PROLIFERATING CELL FACTOR genes associated with petal variations in zygomorphic flowers of Petrocosmea spp. of the family Gesneriaceae. Plant Physiol. 169, 2138-2151. doi: $10.1104 /$ pp. 15.01181 
Zhong, J., and Kellogg, E. A. (2015). Duplication and expression of CYC2-like genes in the origin and maintenance of corolla zygomorphy in Lamiales. New Phytol. 205, 852-868. doi: 10.1111/nph.13104

Conflict of Interest Statement: The authors declare that the research was conducted in the absence of any commercial or financial relationships that could be construed as a potential conflict of interest.
Copyright (C) 2016 Huang, Li, Sun, Zhang, Pan, Cheng, Wang and Zhang. This is an open-access article distributed under the terms of the Creative Commons Attribution License (CC BY). The use, distribution or reproduction in other forums is permitted, provided the original author(s) or licensor are credited and that the original publication in this journal is cited, in accordance with accepted academic practice. No use, distribution or reproduction is permitted which does not comply with these terms. 